Preface

\title{
Bleeding and Thrombosis in Patients with Liver Diseases
}

\author{
Ton Lisman, $\mathrm{PhD}^{1}$ Nicolas M. Intagliata, $\mathrm{MD}^{2}$ \\ ${ }^{1}$ Surgical Research Laboratory and Section of Hepatobiliary Surgery \\ and Liver Transplantation, Department of Surgery, University of \\ Groningen, University Medical Center Groningen, Groningen, \\ The Netherlands \\ 2 Division of Gastroenterology and Hepatology, Center for \\ Coagulation in Liver Disease, University of Virginia Medical Center, \\ Charlottesville, Virginia
}

Semin Thromb Hemost 2020;46:653-655.

Patients with liver disease acquire substantial changes in their hemostatic system. While historically these changes were interpreted as indicating a bleeding tendency, we now understand that patients with liver disease-associated hemostatic changes do not have a "coagulopathy." Rather, the simultaneous decrease in pro- and antihemostatic drivers results in a rebalanced hemostatic status. ${ }^{1,2}$ It has been postulated that the fragile hemostatic balance can easily be tipped toward a hypo- or hypercoagulable state, which explains the occurrence of both bleeding and thrombotic complications. However, a substantial proportion of bleeding complications in patients with cirrhosis are likely unrelated to hemostatic failure but rather relate to portal hypertension or mechanical injury to vessels, for example, in variceal bleeding and laceration of vessels during invasive procedures. Thus, over the last two decades, the hemostatic concerns in patients with liver diseases have shifted from a real concern for bleeding and major attempts to improve hemostasis by prophylactic use of blood products and other hemostatic agents to a concern for thrombosis, with increasing attention for the optimal use of anticoagulant therapy. $^{3-7}$

In this issue of Seminars in Thrombosis and Hemostasis, we highlight new developments in the broad context of the prevention and management of bleeding and thrombosis in patients with liver diseases. Although, unfortunately, highquality clinical evidence to guide hemostatic management is still lacking, there is accumulating lower quality evidence and increasing clinical experience to support the development of rational management advice.

The issue starts with a contribution of Roberts and Bernal who review the magnitude of bleeding and thrombotic complications in patients with acute and chronic liver failure, with a focus on spontaneous bleeding and bleeding associat-

Address for correspondence Ton Lisman, PhD, Department of Surgery, University Medical Center Groningen, BA33, Hanzeplein 1, 9713 GZ Groningen, The Netherlands (e-mail: j.a.lisman@umcg.nl).

ed with invasive procedures including liver transplant surgery. ${ }^{8}$ The authors emphasize that bleeding complications in patients with cirrhosis are common but often appear unrelated to the coagulopathy of liver disease. Also, the challenges in the prevention and treatment of thrombotic complications such as deep vein thrombosis, pulmonary embolism, and portal vein thrombosis are discussed. Subsequently, Northup and Davis discuss shortcomings in clinical studies on the prevention and management of bleeding and thrombosis and provide recommendations for future studies. ${ }^{9}$ The authors stress the importance of clear and validated clinical end points, proper sample sizes, and multicenter and multidisciplinary efforts to take this field forward. Next, NicoarăFarcău et al discuss new insights into the pathogenesis and management of cirrhotic portal vein thrombosis. ${ }^{10}$ Portal vein thrombosis is a common complication of cirrhosis, but whether (asymptomatic) portal vein thrombosis is just a "predictable milestone" of cirrhosis or an active driver of disease progression continues to be debated, and, hence, if and who qualifies for active treatment is not fully established. The authors summarize their approach and indicate areas in which additional studies are required. A paper by Khemichian and Terrault discusses the use of thrombopoietin receptor agonists in patients with cirrhosis. ${ }^{11}$ Although there is increasing consensus that the correction of a prolonged international normalized ratio in patients with cirrhosis prior to procedures is not required, there is no consensus on how to handle cirrhotic thrombocytopenia in the context of procedures. Khemichian and Terrault review evidence on efficacy and safety of thrombopoietin receptor agonists in cirrhosis and demonstrate that these agents effectively increase platelet count without major side effects, but there is little evidence that they reduce procedural bleeding.

Copyright $\odot 2020$ by Thieme Medical Publishers, Inc., 333 Seventh Avenue, New York, NY 10001, USA. Tel: +1(212) 760-0888.
DOI https://doi.org/

10.1055/s-0040-1715453. ISSN 0094-6176.
Diseases; Guest Editors: Ton Lisman, PhD, and Nicolas Intagliata, MD. 
The next set of papers deal with laboratory studies that are commonly used to estimate hemostatic capacity in patients with liver disease. Lebreton et al summarize what we have learned from using thrombin generation tests in patients with liver diseases and indicate issues with this methodology..$^{12}$ The authors provide recommendations for standardizing future studies in this area and indicate a set of analytical and preanalytical parameters that need to be reported in published work to be able to compare the results of different studies. While thrombin generation tests are still a research-type assay, viscoelastic tests such as TEG (thromboelastography) and ROTEM (rotational thromboelastometry) are widely used in clinical practice to guide the hemostatic management of patients with liver diseases. Janko et al summarize the current use of these tests in clinical practice and indicate limitations of these assays. ${ }^{13}$ Next, Stotts et al outline hemostatic changes in patients with liver disease, with a focus on the wide clinical spectrum of cirrhosis. ${ }^{14}$ The authors argue that many published data are in mixed cohorts of patients with differing etiology and severity of disease, and propose that future studies should aim to dissect the varying severities, with a special focus on isolating patients with acute-on-chronic liver failure, which is a unique clinical syndrome with unique hemostatic features.

The final set of papers discuss specific novel developments in the field. First, von Meijenfeld and Jenne discuss the roles of neutrophil extracellular traps (NETs) in liver diseases. ${ }^{15}$ NETs are weblike structures expelled by neutrophils consisting of DNA and DNA-binding proteins. While NETs are crucial in host defense and contribute to pathogen clearance within the liver, NETs can also do harm. Specifically, NETs have been implicated in the progression of various types of liver disease and have been shown to activate hemostasis within the liver, which may lead to harmful micro- and macrovascular clot formation within and around the liver. Possibilities and limitations of testing for NET formation in clinical samples are also discussed. Next, Starlinger et al discuss the role of hemostatic components in liver regeneration. ${ }^{16}$ Surgical removal of part of the liver initiates a regenerative response that is modulated substantially by both platelets and fibrin that are deposited within the regenerating liver. Studies from animal models and clinical observations are consistent with a complex interplay of platelets and fibrin generation driving liver regeneration after partial hepatectomy. This evidence and the knowns and unknowns of the exact molecular mechanisms underlying hemostasis-assisted liver regeneration are reviewed in this paper. In a subsequent study, Karangwa et al discuss challenges in hemostatic management during ex-vivo perfusion of human donor liver prior to transplantation. ${ }^{17}$ Historically, donor organs are kept on ice from procurement until transplantation. However, in recent years, machine perfusion of donor organs with the aims to assess graft quality and to resuscitate the organ has been introduced in clinical practice. When livers are perfused at (sub)physiological temperatures, synthesis of proteins (including coagulation factors) starts, and these de novo produced proteins are released into the perfusion fluid.
Karangwa et al show that this leads to the production of physiologically relevant amounts of coagulation factors, which necessitates anticoagulant strategies to avoid the activation of coagulation with subsequent clot formation within the perfusion circuit. The final paper of this issue deals with the role of portal hypertension in blood loss during liver transplantation. Although, historically, the cirrhotic coagulopathy was held responsible for bleeding during liver transplant surgery, accumulating clinical and biological evidence of the maintenance of hemostatic balance during liver transplantation questions this dogma. Other factors, including the extent of portal hypertension, may be more relevant contributors to perioperative blood loss. Indeed, Arshad et al demonstrate that perioperative blood loss is associated with serum levels of two recently identified markers of portal hypertension. ${ }^{18}$

We hope that readers of this journal enjoy this collection of papers describing new insights in the field of hemostasis and thrombosis in patients with liver disease. This issue is not meant to provide a complete overview of the field, and we refer the reader to numerous recent reviews and book chapters, and a previous liver issue of Seminars in Thrombosis and Hemostasis. ${ }^{19}$

\section{Conflict of Interest}

Dr. Intagliata reports educational grant support from Dova, outside the submitted work.

\section{References}

1 Lisman T, Porte RJ. Rebalanced hemostasis in patients with liver disease: evidence and clinical consequences. Blood 2010;116 (06):878-885

2 Tripodi A, Mannucci PM. The coagulopathy of chronic liver disease. N Engl J Med 2011;365(02):147-156

3 Lisman T, Kamphuisen PW, Northup PG, Porte RJ. Established and new-generation antithrombotic drugs in patients with cirrhosis possibilities and caveats. J Hepatol 2013;59(02):358-366

4 Intagliata NM, Argo CK, Stine JG, Lisman T, Caldwell SH, Violi F; faculty of the 7th International Coagulation in Liver Disease. Concepts and controversies in haemostasis and thrombosis associated with liver disease: Proceedings of the 7th International Coagulation in Liver Disease Conference. Thromb Haemost 2018; 118(08):1491-1506

5 Turco L, de Raucourt E, Valla D-C, Villa E. Anticoagulation in the cirrhotic patient. JHEP Rep 2019;1(03):227-239

6 Intagliata NM, Caldwell SH, Tripodi A. Diagnosis, development, and treatment of portal vein thrombosis in patients with and without cirrhosis. Gastroenterology 2019;156(06): 1582-1599.e1

7 O'Leary JG, Greenberg CS, Patton HM, Caldwell SH. AGA Clinical Practice Update: coagulation in cirrhosis. Gastroenterology 2019; 157(01):34-43.e1

8 Roberts LN, Bernal W. Incidence of bleeding and thrombosis in patients with liver disease. Semin Thromb Hemost 2020;46(06): 656-664

9 Northup PG, Davis J. Shortcomings in design and analysis of clinical studies on bleeding and thrombosis in patients with cirrhosis. Semin Thromb Hemost 2020;46(06):665-672

10 Nicoară-Farcău O, Soy G, Magaz M, et al. . New insights on pathogenesis, risk factors and treatment of portal vein thrombosis in patients with cirrhosis. Semin Thromb Hemost 2020;46 (06):673-681 
11 Khemichian S, Terrault NA. Thrombopoietin receptor agonists in patients with chronic liver disease. Semin Thromb Hemost 2020; 46(06):682-692

12 Lebreton AMD, Sinegre T, Lecompte T, Talon L, Abergel A, Lisman T. Thrombin generation and cirrhosis: state of the art and perspectives. Semin Thromb Hemost 2020;46(06): 693-703

13 Janko N, Majeed A, Kemp W, Roberts SK. Viscoelastic tests (VETs) as point of care tests in the assessment and management of bleeding and thrombosis in liver disease. Semin Thromb Hemost 2020;46(06):704-715

14 Stotts MJ, Lisman T, Intagliata NM. The spectrum of disease severity in cirrhosis and its implications for hemostasis. Semin Thromb Hemost 2020;46(06):716-723
15 von Meijenfeld FA, Jenne CN. Neutrophil extracellular traps in liver disease. Semin Thromb Hemost 2020;46(06):724-734

16 Starlinger P, Luyendyk JP, Groeneveld DJ. Hemostasis and liver regeneration. Semin Thromb Hemost 2020;46(06):735-742

17 Karangwa SA, Lisman T, Porte RJ. Anticoagulant management and synthesis of hemostatic proteins during machine preservation of livers for transplantation. Semin Thromb Hemost 2020;46(06): 743-750

18 Arshad F, Lisman T, Porte RJ. Blood markers of portal hypertension are associated with blood loss and transfusion requirements during orthotopic liver transplantation. Semin Thromb Hemost 2020;46(06):751-756

19 Lisman T, Kwaan HC. Hemostatic dysfunction in liver diseases. Semin Thromb Hemost 2015;41(05):445-446 